\title{
Theory of Static and Dynamic Antiferromagnetic Vortices in LSCO Superconductors
}

\author{
Jiang-Ping Hu and Shou-Cheng Zhang \\ Department of Physics, McCullough Building, Stanford University, Stanford CA 94305-4045
}

\begin{abstract}
A key prediction of the $S O(5)$ theory is the antiferromagnetic vortex state. Recent neutron scattering experiment on LSCO superconductors revealed enhanced antiferromagnetic order in the vortex state. Here we review theoretical progress since the original proposal and present a theory of static and dynamic antiferromanetic vortices in LSCO superconductors. It is shown that the antiferromagnetic region induced by the vortices can be greater than the coherence length, due to the light effective mass of the dynamic antiferromagnetic fluctuations at optimal doping, and close promixity to the antiferromagentic state in the underdoped regime. Systematic experiments are proposed to unambiguously determine that the field induced magnetic scattering originates from the vortices and not from the bulk.
\end{abstract}

\section{Invited Talk at SNS 2001}

$S O(5)$ theory is an unified theory of antiferromagnetism (AF) and superconductivity (SC) in the cuprate superconductors [1].2]. Although AF and SC phases seem to be detached in the experimental phase diagram, this theory predicts a direct transition between them as the chemical potential is varied. As a function of a physical parameter, the direction of the $S O(5)$ superspin vector can be rotated smoothly. However, the chemical potential is hard to control experimentally. Doping in the $\mathrm{AF} / \mathrm{SC}$ transition region is complicated by the chemical and structural inhomogeneities. Therefore, in the $S O(5)$ theory, it was proposed that the interplay between these two phases can be investigated in a controlled way in the vortex state of the superconductor, where the external magnetic field can smoothly rotate the direction of the superspin vector. This theory makes the striking prediction that the core of the vortex is antiferromagnetic [1], in sharp contrast to the metallic vortex core in a conventional superconductor. The AF vortex state (AFVS) can be viewed as a topologically non-trivial texture of the $S O(5)$ superspin vector. Furthermore, it was explicitly proposed that the AF vortex state can be detected in neutron scattering experiments as satellite peaks spaced by $2 \pi / d$, where $d$ is the spacing between the vortex cores [1]. These satellite peaks give a precise experimental definition of the $\mathrm{AF}$ vortex state, where static $\mathrm{AF}$ order coexists with the vortex state of the superconductor.

A theory of the AF vortex core was developed by Arovas et al [3]. This work introduced a method to study the vortex core based on a Schroedinger like equation for the magnetic excitations near the vortex core. Due to the $S O(5)$ constraint on the AF/SC superspin vector [1], the vortex core acts like an attractive potential for the magnetic excitations. If a bound state exists, enhanced low energy magnetic fluctuation is localized near the vortex core. If the bound state energy goes to zero, static $\mathrm{AF}$ order is established inside the vortex core. Based on estimation of parameters, it was argued that the static vortex core is stable in the underdoped regime [3]. These authors suggested that the AF vortex state can be de- tected in neutron scattering experiments, and predicted that the field induced antiferromagnetic moments should scale with the applied field, or the number of vortices in the system [3]. Soon after this work, Bruus et al [4] analyzed experimental data in YBCO superconductors and argued that the vortex core in optimally doped materials should have enhanced dynamic AF order, with energy below the neutron resonance energy of $41 \mathrm{meV}$. Ogata [5] performed extensive variational calculations on the $t-J$ model and found direct evidence of the static AF vortex core up to doping level of $\delta=0.1$. In a classical Monte Carlo calculation of the $S O(5)$ model, $\mathrm{Hu}$ [6] observed the AF vortex state in the satellite peaks of the AF spin correlation function, which are spaced by $2 \pi / d$. Thermodynamic implications of the AF vortex state has been recently investigated by Juneau et al [7]. Various groups [8, [8, 9, 10] argued that the AF vortex core is expected to lead to strong suppression of the local density of states, consistent with the STM experiments [11]. Recently, Demler et al [12] described the influence of the superflow kinetic energy and found that this led to an important contribution to the magnetic field dependence of the spontaneous moment in a phase with magnetic long-range order. Furthermore, these authors also developed a theory for dynamic magnetism in the vortex lattice. This approach is reviewed in the same volume by Sachdev [13.

On the experimental side, the search of AF vortex state showed promising results. Early on Vaknin et al 14. found evidence of AF order in the vortex state of YBCO. Katano et al [15] found enhanced AF fluctuations in the vortex state of LSCO. Most striking evidence of AF order in the vortex state is observed recently by Lake et al [16,17]. In the vortex state of optimally doped LSCO superconductors, incommensurate magnetic fluctuations were found in the low energy region inside the spin gap, whose intensity scales with the number of vortices in the system. In the underdoped LSCO superconductors, enhanced static AF order was discovered in the vortex state. In another related system, $\mathrm{La}_{2} \mathrm{CuO}_{4+y}$ [18, 19, similar 
field induced enhancement has been discovered.

The purpose of this paper is to show that the theory of the $\mathrm{AF}$ vortex state [1, 3] can be applied straightforwardly to explain the recent neutron scattering measurements by Lake et al 16, 17. We discuss various energy and length scales in the problem, and show that experiments are performed in a regime where static and dynamic AF vortex state can be observed. In the original theory, only the case of the commensurate AF fluctuations were discussed. As we shall see, the theory can be extended in a simple way to accommodate incommensurate AF fluctuations as well. Most importantly, we show that one can determine unambiguously whether the field enhanced $\mathrm{AF}$ fluctuation originates from the bulk or from the vortices, and show that present experiments already provides very strong evidence that the later case is realized.

The starting point of our consideration is the effective $S O(5)$ theory of the magnetic fluctuations linearized in an inhomogeneous background of the SC condensate [1, 3]. This theory can be cast into the following form of the effective Lagrangian $\mathcal{L}=\frac{1}{2 \Delta_{s}}\left(\partial_{t} m_{\alpha}\right)^{2}-H$, where $m_{\alpha}(x, t)$ is the local $\mathrm{AF}$ order parameter and

$$
H=\frac{1}{2} \sum_{p} \Lambda_{p} m_{\alpha}(p) m_{\alpha}(-p)+\frac{1}{2} \sum_{x} v(x) m_{\alpha}(x)^{2}
$$

where $\omega=\sqrt{\Lambda_{p} \Delta_{s}}$ is the dispersion of the AF fluctuation in the absence of the external magnetic field. In the LSCO superconductors, we can approximate it with the following form:

$$
\Lambda_{p}=\Delta_{s}+\frac{\left(p-Q_{0}\right)^{2}}{2 M^{*}}
$$

Here $\Delta_{s}$ is the spin gap energy. In optimally doped LSCO superconductors, $\Delta_{s} \approx 7 \mathrm{meV}$. $Q_{0}$ is the wave vector of the incommensurate AF fluctuations in LSCO [20], which can be interpreted as dynamic stripes [21,22]. $M^{*}$ is the effective mass for the AF fluctuations, and we shall give an estimate of this parameter later. $v(x)$ is the potential due to the SC order parameter. In the $S O(5)$ theory, there is a constraint between the $\mathrm{AF}$ and $\mathrm{SC}$ order parameters in the form $m_{\alpha}^{2}+|\psi|^{2}=1$, where $\psi$ is the $\mathrm{SC}$ order parameter. If we implement this constraint by a soft spin constraint, of the form $-g\left(m_{\alpha}^{2}+|\psi|^{2}\right)+u\left(m_{\alpha}^{2}+|\psi|^{2}\right)^{2}$, we see that a repulsive coupling between the two order parameters is implied. This would remain true in the presence of a $S O(5)$ symmetry breaking term $w m_{\alpha}^{2}|\psi|^{2}$, as long as $w>-2 u$. The repulsion between the $\mathrm{SC}$ and the AF order parameters is the crucial physics for all field induced magnetic phenomena. If these two order parameters were decoupled 23], no such effects would exist. Linearizing with respect to this repulsive coupling in the bulk we obtain the spin gap $\Delta_{s}$. However, at the center of the vortex core, the SC order parameter vanishes, and the effective spin gap is lowered. Therefore, there is an effective attractive potenial $v(x)$ for the AF magnetic fluctuations inside the vortex core. We assume that it takes the form

$$
v(x)=\sum_{i} v_{0}\left(x, x_{i}\right), \quad v_{0}\left(x, x_{i}\right)=-V e^{-\frac{\left(x-x_{i}\right)^{2}}{2 \xi^{2}}}
$$

where $V<\Delta_{s}, \xi$ is the SC coherence length and $x_{i}$ describes the center of the vortices. Equations (1), (2) and (3) determines the AF fluctuation spectrum in the vortex state.

The description of the free propagation of the AF magnetic fluctuation obtained from the linearized $S O(5)$ theory is similar to that first obtained from analytically continuing a magnon triplet from a quantum disordered state, such as the spin Peierls state [24,25]. However, the crucial point here is the attractive interaction between the AF magnetic fluctuations with the vortices, which is first obtained from the $S O(5)$ theory. In the original theory, a commensurate form of the dispersion is assumed. However, it was later found that competing interactions in the $S O(5)$ theory could produce dispersion in the form of (2), with a general incommensurate wave vector $Q_{0}$ [26]. For our current discussion, it is easy to see that $Q_{0}$ can be trivially gauged away from the problem. If we perform a phase transformation

$$
m_{\alpha}(x)=e^{i Q_{0} x} n_{\alpha}(x)
$$

we see easily that the quadratic Hamiltonian can be diagonalized by the following Schroedinger like equation for $n_{\alpha}(x)$ :

$$
\left(-\frac{\nabla^{2}}{M^{*}}+v(x)\right) n_{\alpha}(x)=\lambda n_{\alpha}(x)
$$

with energy given by $\Delta_{s}+\lambda$. This equation was first introduced in equation (10) of ref. [3], and forms the basis for subsequent studies on AF order in the vortex state.

Equation (5) describes the motion of an effective quantum particle with mass $M^{*} / 2$ in a periodic array of attractive vortex centers. The important physics here is that in $\mathrm{D}=2$, at least one bound state forms inside the attractive potential. This bound state for the collective $\mathrm{AF}$ fluctuation is not to be confused with the bound state of quasi-particles inside the vortex cores. Let us first consider the simple case where only one bound state forms inside the attractive potential, with energy $E_{B}$. The localization length of the bound state can be estimated to be

$$
l \sim \sqrt{\frac{\hbar^{2}}{M^{*}\left(\Delta_{s}-E_{B}\right)}}
$$

In the limit where $l<d$, the band width of the vortex band can be estimated to be

$$
t \sim V e^{-d / l}
$$


The three length scales, the SC coherence $\xi$, the AF magnetic localization length $l$, the inter-vortex spacing $d$, and the four energy scales, the spin gap $\Delta_{s}$, the attractive potential $V$, the bound state energy $E_{B}$ and the vortex band width $t$ are illustrated in Fig. 1. The concept of a static or dynamic AF vortex core is a sharply defined concept. If there is no bound state, then there is no sense in which one can define the concept of AF vortex core. However, if bound states exists inside the spin gap, the vortex core develops dynamic $\mathrm{AF}$ ordering, with well defined ordering energy scale $\Delta_{s}-E_{B}$ and well defined ordering length $l$. One can view $l$ as the size of the vortex and due to the finite size quantization, the $\mathrm{AF}$ moment fluctuates dynamically, with time scale $\hbar /\left(\Delta_{s}-E_{B}\right)$. In the regime of weak field, $l<<d$, the bandwidth of the $\mathrm{AF}$ vortex band can basically be neglected. With increasing field, the bound state energy $E_{B}$ decreases, and the inter-vortex coherence $t$ increases as well. When the bottom of the vortex band energy touches zero, the static $\mathrm{AF}$ vortex state is obtained.

With this basic preparation we can now discuss the experimental situation in the optimally doped LSCO superconductors, and present the central argument of this paper that the field induced scattering originates from AF fluctuations localized around the vortex cores. Experiments are done in the weak magnetic field regime where the volume fraction associated with the vortices is extremely small, $f \sim(\xi / d)^{2}<10 \%$. On the other hand, the field induced magnetic scattering is centered around $4 \mathrm{meV}$, well below the spin gap energy of $\Delta_{s}=7 \mathrm{meV}$. If the field induced AF magnetic fluctuations were extended, scattering by the vortices can only cause an energy shift proportional to $V(\xi / d)^{2}$, which vanishes in the limit of low vortex volume density. See Fig. 2. In the experimental regime of $f \sim 10 \%$, the energy shift of extended states would be of the order of $\Delta E=V f<$ $\Delta_{s} f<0.7 m e V$, far less than the experimentally observed value of $\Delta E \sim 3 \mathrm{meV}$. Therefore, the Lake et al experiment 116] already shows that it is extremely unlikely that the field induced scattering originates from the bulk. On the other hand, if the field induced AF magnetic fluctuations are localized around the vortices, the energy shift is finite in the limit $f \rightarrow 0$. See Fig. 3. If we assume one bound state per vortex, the field induced intensity $I$ is proportional to $f$. Therefore, this picture predicts that the field induced intensity is directly proportional to $H / H_{c 2}$. These are the precise signatures of the AF vortex core in the limit of low vortex volume density. In Fig. 2 of ref. 16, one sees clearly that the continuum above the spin gap is little changed by the field, but new spectral weight is introduced with a centroid well detached from the continuum. The experimental fact that the centroid of the field induced signal is well below the spin gap and the intensity is proportional to $f$ provides strong evidence that the field induced scattering is localized around the vortices. A more systematic analysis should plot both the energy centroid and the intensity of the field induced signal as a function of $f$. Since $t$ depends exponentially on $d$, it vanishes faster than any other energy scales in the limit of $f \rightarrow 0$. Therefore, if $E_{B} \rightarrow$ finite and $I \propto f$ in the extrapolated limit of $f \rightarrow 0$, unambiguous evidence in support of the AF vortex core can be established. The $f \rightarrow 0$ limit is an useful tool to label the bands, since once $f$ becomes finite, there is no rigorous distinction between the extended and localized bands.

Considerations of the energy of the field induced scattering leads to the conclusion that the they must be localized near the vortex cores. Let us now fix the energy at $\Delta_{s}-E_{B}$ and consider the spatial distribution of the AF fluctuations. The three length scales can be easily estimated from the experiments, giving $\xi \sim 4 a_{0}, l \sim 20 a_{0}$ and $d \sim 40 a_{0}$. Here $l$ is estimated from the width of the momentum distribution function at fixed energy of $4 \mathrm{meV}$. Theory predicts [1,6, 6 , 22 that the momentum distribution function for the $n(x)$ field consists of a series of satellite peaks spaced by $G=2 \pi / d$, centered around the main peak at $q=0$, where the intensity of each satellite peak is covered by an envelope function of the width $1 / l$. See figure (何). Neutron scattering couple to the

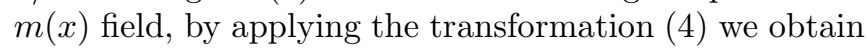
the same pattern, but with the center of the envelope function shifted to incommensurate wave vector $Q_{0}$. See figure (5). Since the momentum shift, or gauge, transformation (4) applies equally to both extended and localized states, the AF fluctuation localized near the vortex core is associated with the exactly the same incommensurate wave vector $Q_{0}$ as the AF fluctuations in the bulk.

Now we need a quantitative argument on why the observed AF coherence length $l \sim 20 a_{0}$ seems longer compared to $\xi$. From equation (6), we see that this could be due to the light effective mass $M^{*}$. But $M^{*}$ could be independently measured in the neutron scattering experiment in the absence of the applied field, by fitting the experimentally measured AF dispersion with $\omega_{p}=\sqrt{\Lambda_{p} \Delta_{s}}$ given in equation (2). This provides an independent consistency check on our theory. A small effective mass translates into steep dispersion in $\omega_{p}$ versus $p$. Experimentally, the incommensurate peak of the momentum distribution function for various energies does seem to shift, implying a very small effective mass. If we assume that the incommensurate peak does not shift over an energy range of $8 \mathrm{meV}$, and momentum shift less than $\left(10 a_{0}\right)^{-1}$ can not be detected, we obtain a value for $M^{*}$ which is roughly consistent with the coherence length $l \sim 20 a_{0}$. It would be highly desirable to improve the experimental accuracy and quantitatively check the relation between the inelastic neutron scattering in the absence of the field, bound state energy and the coherence length in the vortex state.

As the external magnetic field increases, $d$ decreases and the intervortex coherence $t$ increases. For $d \sim l$, it ceases to take the form $t \sim V e^{-d / l}$. A similar effect 
arises when the system is more underdoped, where both $\xi$ and $l$ increases, resulting in the increase of $t$. The local bound state near each vortex now form a band, with band width $t$. As a function of the applied field, the center of the band shifts downwards, and the bandwidth increases. Within Born scattering, the shift of the band minimum can be estimated to be

$$
E_{\text {min }}=E_{B}-\alpha V f
$$

where $\alpha$ is a dimensionless constant. When the bottom of this vortex band touches zero, the system makes a phase transition into a AF vortex state as predicted in [1, 3]. However, Demler et al 12] observed that in this regime, the superflow makes an important correction to the field dependence, and find a logarithmic correction 17, 19. As argued in previous paragraphs, the static AF moments are not localized in a region of the size of $\xi$, but of the size of $l$, which is now comparable to $d$. It is important to point out here that due to the repulsion between $\mathrm{AF}$ and $\mathrm{SC}$ order parameters, the static AF order near the vortices is always larger than the AF order in the bulk. However, these quantitative corrections do not change the basic periodic structure. As pointed out in ref. [1, 6, 12], this periodic pattern can be detected directly in neutron scattering experiments as satellite peaks. One should observe the momentum distribution functions displayed in Fig. 2 and 3, for quasi-elastic scatterings. These satellite peaks provide direct experimental evidence for the static $\mathrm{AF}$ order in the vortex state. Recent neutron scattering experiments in underdoped LSCO superconductors revealed long ranged static incommensurate $\mathrm{AF}$ order in the presence of an external magnetic field [17,19, where the field induced signal is proportional to $\bar{H} / H_{c 2}$, or the number of vortices in the sample. It is highly desirable to refine the momentum resolution in the experiments and detect the satellite peaks directly.

Now we are in a position to understand why relatively moderate field $H_{A F V S}$ can induce a transition into a statically ordered AF vortex state. If there were no localized states near the vortices in the $f \rightarrow 0$ limit, the transition to a statically ordered state arises from the band of extended states, whose bottom touches zero. However, if the band of localized state exists below the extended band, its bottom will always touch zero first. Since bands are not expected to cross as the external magnetic field is varied, we can roughly estimate the ratio between these two critical fields to be $H_{A F V S}($ bound $) / H_{A F V S}($ extended $) \sim\left(\Delta_{s}-E_{B}\right) / \Delta_{s} \sim$ $1 / 2<1$. In other words, transition due to the localized band always preempts the transition due to the extended band. From this argument, we reach the important conclusion that the relatively moderate field can induce a transition into a statically ordered AF vortex state precisely because the AF order originates from the vortices.

In above discussions we mostly focused on neutron scattering experiments in LSCO. However, the theory is generally applicable to all high $T_{c}$ materials. In recent NMR experiments [27], enhanced AF fluctuations around the vortex cores have been detected in the YBCO system. In a recent STM experiment, Hoffman et al 28] detected CDW-like order around the vortex core in the BSCO system. In both cases, the magnetic field induced order are well localized around the vortex core, with a localization length greater than the vortex core itself. The important point to be stressed here is that the localization length is finite, in accordance to the general discussions outlined above. It would be highly desirable to extend the neutron scattering experiments under the magnetic field already performed by Dai et al [29] on YBCO systems to higher field, and to look for the dynamic AF bound state around the vortex core, with bound state energy of few $m e V$ below the neutron resonance energy.

Finally, we would also like to comment on a new order parameter which emerges in the AF vortex state. In ref. [1], one of us introduced a general constraint between the $S O(5)$ superspin order parameters $n_{a}$ and the $S O(5)$ symmetry generators $L_{a b}$, which takes the form:

$$
\epsilon^{a b c d e} n_{c} L_{d e}=0
$$

In regions of space where both the $\mathrm{AF}$ order parameter $n_{2,3,4}$ and the SC order parameters $n_{1,5}$ coexist, the $S O(5)$ constraint (9) automatically implies that the corresponding $\pi$ operators $L_{12,13,14}$ and $L_{25,35,45}$ would acquire non-zero expectation values as well. Therefore, in the region $\xi<r<l$ around the vortex core, where both $\mathrm{AF}$ and $\mathrm{SC}$ orders coexist, the order associated with the $\pi$ operators in the $S O(5)$ theory exists as well. In a subsequent paper, we shall fully explore the experimental consequence of this observation.

In conclusion we have reviewed theoretical progress since the original proposal of AF order in the SC vortices [1],3], and presented a semi-quantitative theory of the static and dynamic AF order in the vortex state of LSCO superconductors. It is argued that the field induced signals found in experiments arise from the dyanmic and static AF order in the superconducting vortices. We showed that the intermediate length scale $l$ describes the localization of AF order near SC vortices. This length scale is large in the LSCO materials because the effective mass for the incommensurate AF fluctuations in the absence of the field is anomalously small. However, this length scale is finite in the limit of low vortex density. Whether the field induced scattering originates from the bulk or from the vortices can be systematically distinguished in neutron scattering experiments, where the energy shift of the field induced scattering is plotted against $H$, in the limit of low $H$. If the energy shift remains finite in the zero field limit, an unambiguous case for the AF vortices can be established. Satellite peaks are predicted in the momentum distribution functions for both quasi-elastic and inelastic scatterings, revealing the static 
and dynamic AF order of vortices. An exact argument based on gauge transformation is presented to show that the AF order localized around the vortices has the same incommensurate wave vector as the bulk AF fluctuations.

The theoretical prediction and experimental discovery of the AF vortex state offers crucial insights into the microscopic mechanism of high $T_{c}$ superconductivity. Various theoretical proposals differ only in the nature of the intermediate state between the $\mathrm{AF}$ and the $\mathrm{SC}$ state. If doping or chemical potential were the only route connecting these two phases, the problem of chemical inhomogeneities will always obscure the underlying physics. The external magnetic field provides a clean alternate route to connect these two phases and a new route to attack this problem. Unlike the case of chemical doping, experiments, coupled with the scaling arguments presented in this work, can precisely determine the real space locations of the AF signals. While the $S O(5)$ theory predicts an $\mathrm{AF}$ vortex state [1], other theories predict $h c / e$ vortex [30], visons [31] and staggered flux phase [32]. Therefore, the nature of the vortex state is important in distinguishing among the various theoretical proposals.

We would like to acknowledge useful discussions with Drs. G. Aeppli, S. Davis, E. Demler, W. Hanke, W. Halperin, S. Kivelson, Y. Lee and S. Sachdev for useful discussions. This work is supported by the NSF under grant numbers DMR-9814289. JP Hu is also supported by the Stanford Graduate fellowship.

[1] S. C. Zhang, Science 275, 1089 (1997).

[2] S. C. Zhang et al., Phys. Rev. B 29, 3721 (1999).

[3] D. Arovas, J. Berlinsky, Kallin, and S. C. Zhang, Phys. Rev. Lett. 79, 2871 (1997).

[4] H. Bruus, K. Eriksen, M. Hallundbk, and P. Hedegard, Phys. Rev. B 59, 4349 (1998).

[5] M. Ogata, Int. J. Mod. Phys. B 13, 3560 (1999).

[6] X. Hu, cond-mat/9906237.

[7] M. Juneau et al., cond-mat/0106172 .

[8] B. M. Andersen, H. Bruus, and P. Hedegard, condmat/9906233 .

[9] J. H. Han and D. H. Lee, cond-mat/0003222 .

[10] M. Franz and Z. Tesanovic, Phys. Rev. B 63, 64516 (2001).

[11] C. Renner et al., Phys. Rev. Lett. 80, 3606 (1998).

[12] E. Demler, S. Sachdev, and Y. Zhang, Phys. Rev. Lett. 87, 067202 (2001).

[13] S. Sachdev, cond-mat/0108238 .

[14] D. Vaknin, J. Zarestky, and L. Miller, Physica C 329, 109 (2000).

[15] S. Katano et al., Phys. Rev. B 62, R14677 (2000).

[16] B. Lake et al., Science 291, 1759 (2001).

[17] B. Lake et al., cond-mat/0104026 .

[18] B. O. Wells et al., Science 277, 1067 (1997).
[19] B. Khaykovich et al., unpublished .

[20] J. Tranquada et al., Nature 375, 561 (1995).

[21] J. Zaanen and O. Gunnarsson, Phys. Rev. B 40, 7391 (1989).

[22] V. J. Emery and S. Kivelson, Physica 235, 189 (1994).

[23] A. Aharony, cond-mat/0107585 .

[24] A. V. Chubukov, S. Sachdev, and J. Ye, Phys. Rev. B 11, 919 (1994).

[25] S. Sachdev, cond-mat/0108238.

[26] J. Hu and S. Zhang, Phys. Rev. B 64, R100502 (2001).

[27] V. F. Mitrovic et al., cond-mat/0108293 .

[28] J. E. Hoffman et al., unpublished .

[29] P. Dai et al., Nature 406, 965 (2000).

[30] S. Sachdev, Phys. Rev. B 45, 389 (1992).

[31] T. Senthil and M. P. A. Fisher, Phys. Rev. Lett. 86, 292 (2001).

[32] J. Kishine, P. A. Lee, and X. G. Wen, cond-mat/0103148

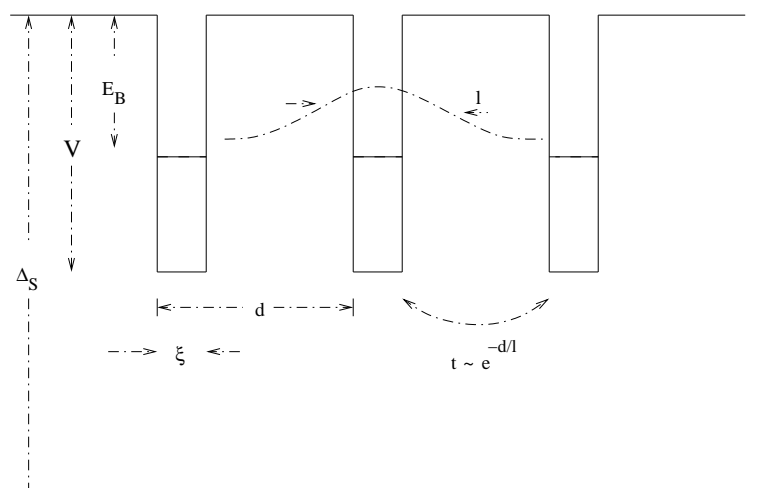

FIG. 1. Illustration of the energy and length scales in the problem. $\Delta_{s}$ is the spin gap energy, $V$ is the attractive potential due to $\mathrm{SC}$ vortices, $E_{B}$ is the bound state energy and $t$ is the bandwidth of the AF vortex band. $\xi$ is the SC coherence length, $l$ the localization length of AF fluctuations around the vortices and $d$ is the inter-vortex spacing.

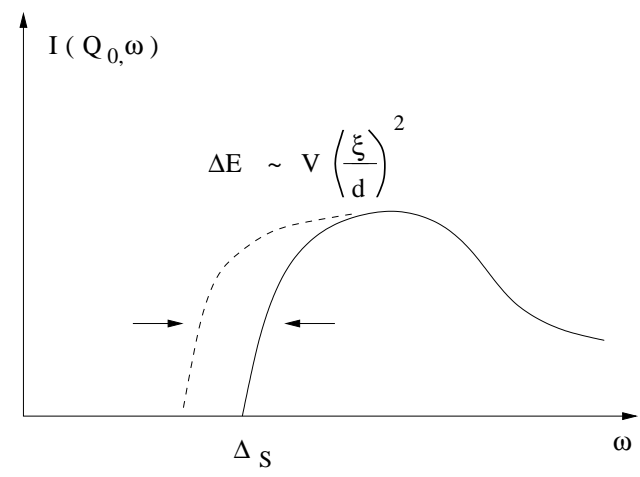

FIG. 2. If no bound states exist, energy of extended states can only shift by an amount of the order of $V(\xi / d)^{2}$, which is vanishingly small in the limit of $d \rightarrow 0$. 


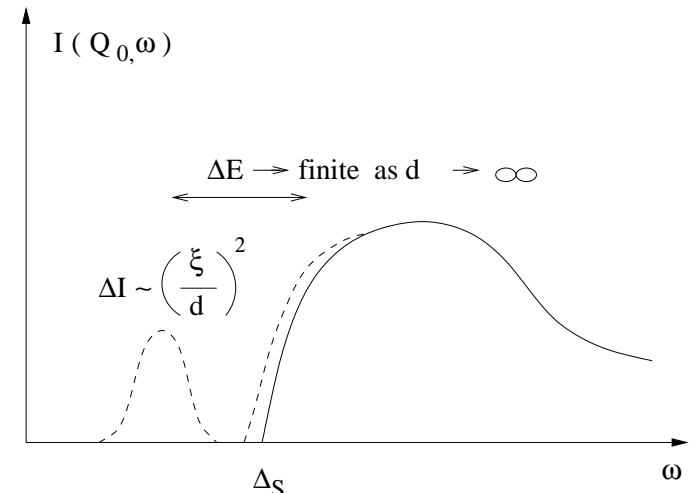

FIG. 3. In the presence of bound states, the energy shift is finite, while the intensity due to the bound state band is proportional to $(\xi / d)^{2} \sim H / H_{c 2}$.

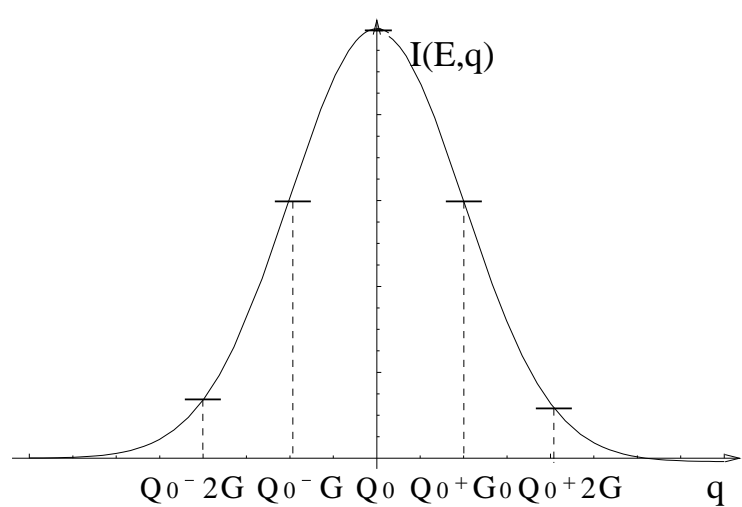

FIG. 4. At energy $E=\Delta_{s}-E_{B}$ for optimal doping and $E=0$ for underdoping, the momentum distribution function consists of a series a delta function peaks, spaced by $G=2 \pi / d$. The envelope of these peaks forms a broader peak centered around the incommensurate wave vector $Q_{0}$, with width $1 / l$.

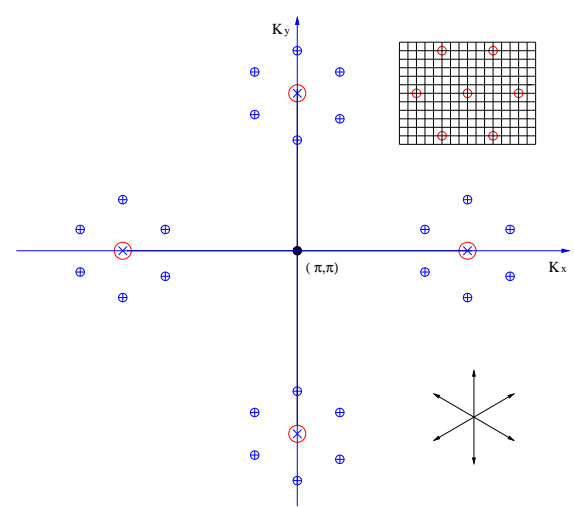

FIG. 5. A more explicit illustration of the satellite peaks near the four incommensurate spots. The inset shows the vortex lattice in real space. 